\title{
Concept Measurement in Alcohol Serving Establishment Research
}

\author{
Rebecca K. Murray \\ College of Arts and Sciences, Creighton University, Omaha, USA \\ Email: rebeccamurray@creighton.edu
}

How to cite this paper: Murray, R. K. (2018). Concept Measurement in Alcohol Serving Establishment Research. Advances in Applied Sociology, 8, 1-24.

https://doi.org/10.4236/aasoci.2018.81001

Received: October 28, 2017

Accepted: January 7, 2018

Published: January 9, 2018

Copyright (C) 2018 by author and Scientific Research Publishing Inc. This work is licensed under the Creative Commons Attribution International License (CC BY 4.0).

http://creativecommons.org/licenses/by/4.0/

\begin{abstract}
This research critically examines traditional quantitative measurements of alcohol establishments and assaults. In doing so, the research first performs a quantitative, spatially-lagged regression model measuring the relationship between location of alcohol establishment and assaults, using traditional measurements of liquor license designation and reports to the police. It then examines the same phenomenon using qualitative measurements. This includes creating a designation of "criminogenic" establishments through atmosphere assessments and employee perception of assault. Results indicate that qualitative analysis, while not wildly divergent in results, may allow more nuanced operationalization of some of the concepts suggested in Routine Activity Theory. The implications of these definitions are considered in terms of their probable impact on previous research results, and future direction in developing accurate measurement in this area is discussed.
\end{abstract}

\section{Keywords}

Spatial Analysis, Mixed Methods, Alcohol Research

\section{Introduction}

Criminology has exploded in recent years with increasingly complex quantitative research methods, allowing the inclusion of larger sample sizes, and more sophisticated models than those seen just a few years ago. In 2010, special edition of Journal of Criminal Justice Education was dedicated to the latest of the specialized and sophisticated quantitative research methods available to our discipline (Volume 21, Number 2; June 2010). Here, guest editor Travis Pratt notes in his introduction that "The qualitative-quantitative dichotomy strikes many as, at best, an empty debate, and at worst, utterly foolish-much like debating whether a hammer or monkey wrench is 'better"', (p. 103). His point is well-taken, but as 
the issue then goes on to describe various quantitative techniques such as groupbased trajectory models (Nagin \& Piquero, 2010); spatial modeling (Fornango, 2010); structural equation modeling (Gau, 2010) and the like. This suggests that in fact, quantitative research methods do continue to take center-stage when it comes to criminological research.

Obviously, there are many benefits to quantitative analysis, and particularly analysis such as advanced spatial modeling has allowed researchers to rapidly advance urban research. However, a crucial component often overlooked in the "qualitative/quantitative" debate, and frankly, often taken for granted in many advanced quantitative studies, is that of appropriate concept measurement. The current research, rather than weighing in on the "qualitative versus quantitative" debate, aims to show that type of analysis can seriously affect measurement and, as such, the precision with which criminal justice research uncovers knowledge.

Criminological research, and especially that concerning urban data, often focuses on the amount of available data, rather than asking whether the appropriate measurement techniques have been used to create this data. The current research takes a specific approach to demonstrating the various problems and issues in measuring concepts of "assaults" and the urban structure of "alcohol serving establishments" in light of Routine Activity Theory. It also introduces some ways in which qualitative methodology may enhance and improve measurement for these concepts.

An examination of the effects of alcohol-selling establishments on crime is relatively complex, and first requires a standard definition of what one means by "alcohol establishment." While other studies have used terms such as "bar" or "tavern", this current research identifies the term more broadly as establishments for which alcohol is legally purchased by patrons to be consumed on the premises. Using traditional measures that incorporate counts of establishments which hold a particular public license may not give an accurate picture of the effects of specific establishments, or serve as accurate measures of theoretical concepts. Thus, this research intends to closely examine the particular nuances of drinking establishments and add to the development of more appropriate measures.

\section{Literature Review}

Routine Activity Theory, as developed by Cohen and Felson (1979), pinpointed three "elements" that precipitated criminal acts: Motivated offenders, suitable targets or victims, and the absence of capable guardians against offenses. Felson (2002) expanded the routine activity approach. He added concepts that identified potential motivations of offenders: 1) props that may have helped produce or prevent a crime, such as weapons or tools, 2) camouflage that helped the offender to have avoided unwanted notice and 3) an audience that the offender wanted to impress or intimidate (Felson, 2002: 22). More than previous work in Routine Activity Theory, Felson's recent work emphasizes environmental influences, such as the effects of place or atmosphere, on criminal acts. 
Felson's (2002) approach to routine activity fits closely with the examination of the effects of non-residential land uses, and particularly with the effects of the presence of alcohol serving establishments on crime. For instance, some of these establishments may involve a casual or even rowdy, atmosphere. In addition, because of the crowded conditions also likely in some of these establishments, not to mention the disregard for safety precautions that may be brought about by intoxication, alcohol serving establishments may provide an arena for hosting probable targets of crime. In the same vein, one might expect other alcohol serving establishments (such as restaurants) to discourage criminal activity through increased guardianship, promotion of an appealing family environment and a strong disapproval of disruptive behavior. Using a Routine Activity Theory perspective, this research looks at the potential of qualitative measurement techniques to explore mechanisms that might encourage violent behavior in particular types of liquor-serving establishments and, conversely, those that might discourage it in others.

\subsection{Crime and Alcohol}

As noted in the introduction, there may be differing definitions surrounding alcohol establishments, but the current research emphasizes the effects of alcohol serving establishments, often referred to as "taverns" "bars" or "pubs" (Graham, Osgood, \& Wells, 2006). However, previous literature does not always differentiate the specific types of establishments as important elements in behavior. In fact, some of the most prominent literature on the subject focuses nearly entirely on the intoxicative effects of alcohol on an individual, rather than the establishment(s) it is consumed in. This research suggests that intoxication affects an individual's decision-making abilities and assesses either propensity to offend or victimization risk based solely on the act of consumption of alcohol and its' biochemical effects. Much of this emphasizes the nature of intoxication on violent behavior. Bye (2007) identified the effect of intoxication on violence between individuals using a time-series analysis. Mitchell, Rutherford, Wrinch, \& Egan (2008) also noted the time-specific effects of alcohol in bar settings over time. Other researchers such as Corbin, et al. (2001) have looked at the effect of alcohol consumption especially by victims on particular crimes such sexual assault. Much of this research looks specifically at the incidence of alcohol use and/or amount of alcohol consumed by an offender or victim as a precursor to violence and/or crime more generally.

As this research focuses on the biochemical effects of alcohol itself, most focus on within-person differences, emphasizing impaired judgment and rationality as well as lowered inhibitions. For the most part, this research has found a significant link between violent incidents and alcohol consumption by one or more parties to that incident (victim and offender). However, more recent research has also indicated that the atmosphere in which intoxication occurs has perhaps as much to do with resulting behavior than the intoxication itself. Hughes, An- 
derson, Morelo, \& Bellis (2008) analyze intoxication as it co-occurs with different environments. Mitchell, et al. (2008) also note the effect of intoxication within particular atmospheres, and how unique interaction with other patrons can play a significant role in potentially problematic behaviors. Greenfield, $\mathrm{Yu}$, Nayak, Bond, Kerr, \& Trocki (2011) also look at consumption by individuals who may perpetrate, or become victims of, assault, but assess intoxicative effects within the broader context of alcohol availability. This type of research explores both intra-personal as well as inter-personal and environmental effects on crime, and fits much better within a Routine Activity framework.

\subsection{Crime and Drinking Establishments}

Another branch of research on alcohol and crime focuses on the availability of alcohol within the larger urban environment. This often utilizes a macro-level view of the places alcohol may be sold and/or consumed, as a part of how environmental factors affect criminal activity. The use of crime mapping has aided significantly in these types studies of crime, and has included assessment of hot spots (See Spring \& Block, 1988 for an overview of hot spots). Researchers such as Costanza, et al. (2001) look very broadly at alcohol density and how it overlaps with crime density in an urban setting. Others, such as Groff (2011) expand this look at spatial density to include a look at how the presence of bars or taverns my influence spaces in close proximity. Some research looks at the influence of alcohol availability on the spatial proximity of particular crimes such as domestic violence (Cunradi, Mair, Ponicki, \& Remer, 2011). Other research consolidates counts to determine concentrations or "hot spots" of liquor availability and crime (see Gorman, Li, \& Horel, 2005).Gruenewald et al., (2006) and Groff (2011) both use sophisticated models that predict the effects of taverns on crime, given differential propensity for crime and alcohol density in various areas of cities. For the most part, strong support has been given to the effects of alcohol availability, or the density of establishments that sell and/or serve alcohol, and crime.

In this, as in other macro-level research, common measurements such as liquor license type are used to measure the presence of "alcohol establishments." More specifically, establishments who are granted a license to both sell and serve liquor are considered alcohol serving establishments (often referred to as bars or taverns) and those who only have licenses to sell are considered offsite liquor establishments (such as liquor stores). In addition, measurements such as police reports have been used to measure "crimes." These reports are created when a crime is reported to law enforcement, but they do not indicate whether a person was actually prosecuted. Most of the large-scale studies on alcohol availability use these or similar measures for the concepts of crime and alcohol availability. While these standardized definitions are not only common but necessary in macro-level criminological research, it is important to note that they make important assumptions which may not necessarily be accurate. Common measurement techniques for alcohol outlets assume that all establishments who share similar liquor licenses (whether "onsite" or "offsite") share an atmosphere 
with each other. Common measurements of crime, particularly those which use reports of crime, also assume that those who notify police share a common definition of what is in fact "criminal".

When examining the effects of alcohol availability and crime, the idea that not only what or how much one consumes has an effect on one's behavior, but also the environments where it is purchased, consumed, or both, is important. Parker (1995) suggests that the process by which violence occurs varies among individuals according to imbibitions and on the sociological aspects of the drinking situation. When examining the effects of bars or other liquor-selling establishments, alcohol consumption is mixed with the particular social or environmental atmosphere of the place in which alcohol is purchased and/or consumed.

\subsection{Alcohol Culture and Criminal Events}

A relatively recent and smaller area of research on crime and alcohol has focused more specifically on the culture of the places within which alcohol is consumed. This research is often more in-depth and nuanced, noting the difference between the culture of different alcohol-serving establishments. Quigley, Leonard, \& Collins, (2003: 756) identify the notion of "violent bars" as particular establishments that attract violence from patrons. Buddie \& Parks (2003) note the same idea of violent context on risk specifically for women. Graham, Bernards, Osgood, Homel, \& Purcell, (2005) specify the role that staff might play in forming of bar or tavern culture, and even affecting whether it creates a benign or aggressive atmosphere. This type of research is unique in that it tends to utilize qualitative methods such as observations (such as in the case of Graham, et al., 2005) and interviews (which is the case in Buddie \& Parks, 2003; Quigley, et al., 2003) to better determine the factors that coincide both with the existence of alcoholserving establishments and the intoxicative effects of alcohol itself.

While all areas of research on alcohol and crime have been invaluable in increasing knowledge of the alcohol/crime link, only that which seeks to describe the culture of alcohol establishments takes into account the implications of the immediate environment on criminal events. As such, this branch seems to fill a gap presented by both biochemical and geographical bar research. Studies focusing on biochemical effects of alcohol overlook the potentially aggravating environments in which alcohol is often consumed, while macro-level research tends over-generalize, assuming a common culture among all alcohol serving establishments.

It is clear that research that has focused on the environments within alcohol serving establishments gives a more complete picture of the complex relationship between alcohol and crime. However, it may also be the case that popular, macro-level quantitative geographical research could potentially be misleading in its results because of definitional assumptions regarding its variables that are not verified to be accurate. Indeed, it seems that in order to establish a more precise definition of both alcohol outlets and crimes that might take place within 
their confines or vicinity, one must turn to a more in-depth, specific and at least partially qualitative measurement technique.

The current research intends to explore the potential problems inherent in the definitions used in large-scale quantitative alcohol establishment research and explore whether qualitative measurements may be more precise. As such, this endeavor aims not only to show how qualitative research specifically on alcohol serving establishments may be more accurate, but in fact how qualitative research may be used to improve definitional problems inherent in quantitative research. In short, this research is intended to demonstrate the importance of taking a "step back" from the rapid advances of structural techniques and asking if what we are measuring is as well thought-out as how we are measuring the urban structures of alcohol-serving establishments.

\section{Methodology}

This research first performs and then critiques an assessment using large scale spatial count measures. Data for this analysis was obtained from the following sources: The state liquor-licensing agency provided a list of establishments who received a license to sell and serve liquor in the city limits. Police reports were provided by the city police department that identified reports of assault in 2009. Finally, demographic data for individual blocks of the city was downloaded from the United States Census Bureau.

After this macro-level quantitative analysis was completed, an assessment using qualitative techniques was competed on a random sample of establishments in each quadrant of the city. This was done using an atmosphere assessment, which was created using methods from prior research, and interviews with establishment employees.

\section{Quantitative Analysis}

The city of Omaha, Nebraska provided a suitable area for examination because it is a small enough city that all the liquor-serving establishments and felonious assaults were easily obtained and geocoded, but a large enough city that it has a substantial amount of both liquor-serving establishments and felonious assaults. A list of liquor licenses for the city of Omaha was obtained from the Nebraska Sate Liquor Commission. The addresses of these establishments were geocoded using Arc GIS software. The locations were made into a point file and assigned to a particular block. Ninety-eight percent of these addresses were successfully geocoded. A total of 613 establishments had a license to sell liquor to be consumed on premises in 2009. These establishments were spread across 484 blocks, ranging from 1 establishment to 6 (See Table 1).

The current research also uses police incident reports as the "traditional" measure of assaults. In this way, the number of assaults on a particular block in 2009 was determined when the addresses provided in the reports were geocoded and summed for each block. Geocoding of these assaults was successful for $92 \%$ 
Table 1. Distributions of Major Variables-All Blocks.

\begin{tabular}{|c|c|c|c|}
\hline & Value & \# of Blocks & $\%$ of all Blocks \\
\hline \multirow[t]{12}{*}{ Blocks with Assaults ( $\mathrm{N}=1021)$} & 0 & 6412 & 92.30 \\
\hline & 1 & 338 & 4.87 \\
\hline & 2 & 105 & 1.51 \\
\hline & 3 & 29 & 0.42 \\
\hline & 4 & 24 & 0.35 \\
\hline & 5 & 15 & 0.22 \\
\hline & 6 & 5 & 0.07 \\
\hline & 7 & 6 & 0.09 \\
\hline & 8 & 1 & 0.01 \\
\hline & 9 & 4 & 0.06 \\
\hline & 12 & 7 & 0.10 \\
\hline & 13 & 1 & 0.01 \\
\hline \multirow[t]{7}{*}{ Blocks with On-premise Liquor-Serving Est. $(\mathrm{N}=613)$} & 0 & 6463 & 93.03 \\
\hline & 1 & 402 & 5.79 \\
\hline & 2 & 50 & 0.72 \\
\hline & 3 & 23 & 0.33 \\
\hline & 4 & 4 & 0.06 \\
\hline & 5 & 4 & 0.06 \\
\hline & 6 & 1 & 0.01 \\
\hline
\end{tabular}

of the assaults reported. As seen in Table 1, the total number of assaults on all blocks in 2009 was 1021.

A total of eight control variables were used along with the primary independent variable of number of liquor serving establishments on a block in an ordinary least squares regression analysis. ${ }^{1}$ Although, as indicated in Table 1, the distribution was highly skewed for both the dependent variable and the primary independent variable, indicating that OLS regression analysis may not be the most appropriate analysis, it was retained for clarity and ease of explanation. While more substantive research should identify a more appropriate model (such as Poisson/Negative Binomial) to deal well with the skewed distribution, for this research, whose primary purpose was to identify issues with operationalization of theoretical concepts, OLS regression analysis provided a clear and succinct example of quantitative issues.

The control variables were a combination of environmental structure variables and population variables. Five were used to control for any environmental ef${ }^{1} \mathrm{~A}$ t-test was performed as an initial analysis to determine if blocks within Omaha that had one or more bars (as defined by liquor license) also had, on average, more felonious assaults (as defined by police reports) than blocks without liquor-serving establishments. While not reported here, the test showed that blocks with at least one onsite license had an higher average number of assaults. ( $\mathrm{p}<$ $0.0034)$. 
fects from the characteristics of the housing blocks on the city blocks: 1) the number of offsite liquor serving establishments (such as grocery or liquor stores, who sell alcohol to be consumed away from the premises), 2) percent of the housing units on the block that were overcrowded (defined by the Census Bureau as percent of residents living in housing units with 1.01 or more persons per room), 3) percent of structures on the block with ten or more housing units (usually apartments), 4) the block vacancy rate, and 5) area of the block in acres (divided by 10).

Three population variables representing the characteristics of residents of the block were also used as control variables in this research. The included the size of the resident population, the percentage of African American residents, and the percentage of Hispanic residents. As blacks and Hispanics are by far Omaha's largest minorities, representing 13.7 and 13.1 percent of the population, respectively, it was important to control for this population dynamic (U.S. Bureau of the Census, 2010).

Table 2 provides the results of the spatial multiple regression analysis, which gives an indication of the effect of the independent variable of alcohol establishments on the crime of assaults, net of the control variables discussed above. This also indicates that blocks with alcohol-selling establishments did have a statistically significant effect on assaults $(P>C h i s q=0.031)$, although it was not a particularly strong predictor $($ Beta $=0.026)$. This finding in itself differs somewhat from some previous research that found no significant effects for bars (see Costanza, et al., 2001), but was consistent with other research using this traditional measurement technique (see Gorman, et al., 2005).

Spatial analysis takes into account issues of spatial autocorrelation, and that not accounting for this can be problematic in interpreting coefficients (see Ratcliffe, 2005). Statistically, unobserved variables may also be spatially correlated; creating spatial dependence for a dependent variable among, say, contiguous blocks (LeSage, 2002: 7). For the current research, Moran's $I$, a widely used test for spatial autocorrelation (LeSage, 2002) shows that there is positive and signif-

Table 2. OLS Multiple Regression.

\begin{tabular}{cccc}
\hline & Beta & $\mathrm{b}$ & Std. Error \\
\hline On-premise Liquor-serving Establishments & $0.026^{*}$ & $0.047^{*}$ & 0.022 \\
Offsite Liquor-serving Establishments & $0.082^{*}$ & $0.302^{*}$ & 0.045 \\
\% Overcrowded & $0.065^{*}$ & $0.009^{*}$ & 0.002 \\
\% Apartments & $0.048^{*}$ & $0.002^{*}$ & 0.001 \\
Vacancy Rate & $0.095^{*}$ & $0.007^{*}$ & 0.001 \\
Area (per 10 Acres) & -0.009 & 0.000 & 0.000 \\
Population & $0.096^{*}$ & $0.001^{*}$ & 0.000 \\
Percent Black & $0.117^{*}$ & $0.015^{*}$ & 0.001 \\
Percent Hispanic & $0.080^{*}$ & $0.020^{*}$ & 0.002 \\
\hline
\end{tabular}

R-squared: $0.229^{\star} ;{ }^{\star}$ Significant at 0.05 level. 
Table 3. MESS Model (Spatially Lagged).

\begin{tabular}{ccc}
\hline & $\mathbf{b}^{1}$ & Std. Error \\
\hline On-premise Liquor-serving Establishments & $0.183^{\star}$ & 0.022 \\
Offsite Liquor-serving Establishments & $0.120^{\star}$ & 0.045 \\
\% Overcrowded & $0.001^{\star}$ & 0.002 \\
\% Apartments & 0.002 & 0.001 \\
Vacancy Rate & $0.001^{*}$ & 0.001 \\
Area (per 10 Acres) & -0.002 & 0.000 \\
Population & $0.001^{\star}$ & 0.000 \\
Percent Black & $0.030^{*}$ & 0.001 \\
Percent Hispanic & $0.020^{\star}$ & 0.002 \\
\hline
\end{tabular}

R-squared: $0.329^{*}$; ${ }^{\star}$ Significant at .05 level; ${ }^{1}$ Beta is not reported because standardized coefficients are not determined in this model.

icant spatial autocorrelation for felonious assaults (Moran's $I=0.879488 ; \mathrm{p}<$ $0.00001)$. As such, a spatially lagged model was the final quantitative component in assessing the effects of a traditional measurement of bars on assaults.

Table 3 provides results of the spatially lagged analysis, which takes into account not only control variables but also the effects of spatial autocorrelation. The overall $\mathrm{r}$-squared increased from 0.229 to 0.339 , indicating that more variance in assaults was explained while accounting for spatial distribution of the dependent variable. Here, there emerged a significant (though still relatively small) effect for bars, indicating that spatial correlation may have been masking the original effect. All other control variable remained significant except for percent apartments on a block, which lost significance.

Finally, a hot-spot analysis is given in Map 1 identifies the location of the quantitatively measured bars, according to the definition given above, as well as the hot spots of felonious assaults, as measured through kernel density analysis. Standard deviations were used as the break points for gradient variations. As indicated, the while some of the locales of bars overlap with the hot spots of assaults, for the most part, the locations of these establishments are evenly spread throughout the city, and most are not concentrated on or around hot spots of assaults. In other words, the dilution effect of using all establishments seems clear.

\section{Qualitative Assessments}

Because the intent of this research is to comment on the measurements involved in examining the relationship between drinking establishments and violent crime, it is important to understand the definitions given to these establishments. Therefore, an attempt was made to more closely look at the social connection established by drinking alcohol in a designated place. Thus, it was crucial to examine the differences between those establishments that were used primarily for drinking/socializing versus those establishments whose service of 


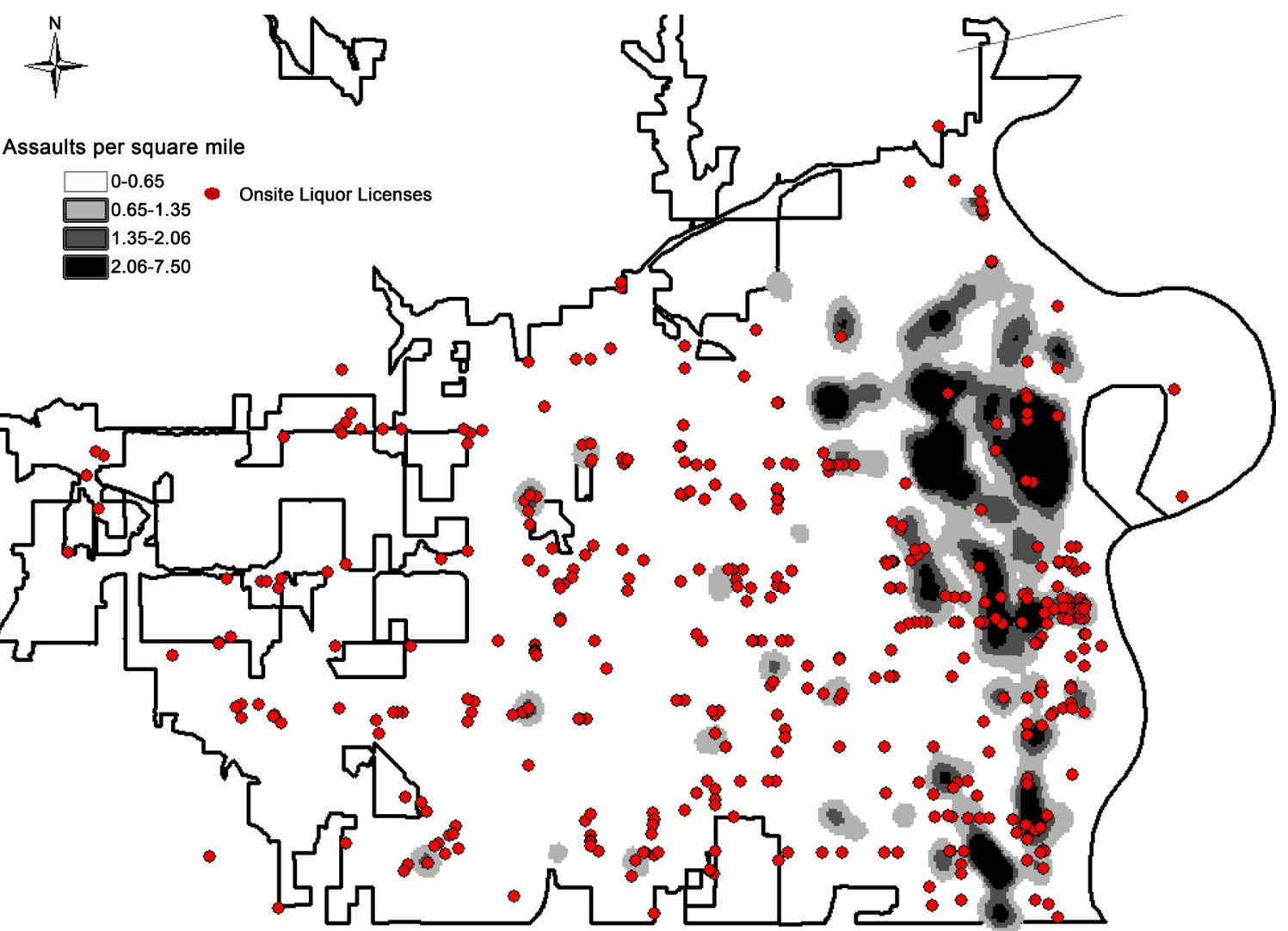

Map 1. Hot Spots of Reported Assaults (2009) and Onsite Liquor Licenses.

alcohol was simply secondary. Distinguishing between these different establishments required a specific assessment of qualities such as the atmosphere of the particular businesses as well as the definition of business purpose, or type of business, as given by their employees.

The other crucial component to assessing definitional validity in current research methodologies was measurement of assaults. Again, utilizing the definitions of employees (primarily wait staff) as to when an assault became a crime (via a police report), the precision of using the traditional method of police reports was examined. Here, we intended to indicate whether a more extensive approach might better determine measurement techniques, both in terms of the identity of urban structures, and in terms of criminal incidents. These "traditional measurements" were critiqued using two qualitative methods: an atmosphere assessment and interviews with employees of alcohol-serving establishments.

\subsection{Atmosphere Assessments}

Following previous research from Graham et al., (2005), a random selection of 40 establishments was chosen from the original list of 613 liquor serving establishments. 10 establishments were randomly selected from each of the four quadrants of the city. Because Omaha is a city which is both racially and culturally segregated, establishments in each of these areas tend to have distinctive charac- 
teristics. Sampling from the city as a whole would oversample from the Southeast quadrant, which has most of the bars in the city, and because the research aimed to identify distinct atmospheres, the decision was made to make an effort to sample throughout the city. Although 40 establishments is a relatively small number (only $6.5 \%$ of the total number of on-premise liquor licenses), the information gleaned from these establishments still makes a strong case for problems with measurement, both for alcohol serving establishments and criminal events. Additionally, this manageable number allowed access to all these establishments, which aided in consistency of the assessment. It is important to note that the small qualitative sample size meant that only a fraction of all establishments were visited, and that this sample was inadequate to generalize results, even to the entire city. However, because this research only intended to demonstrate the inadequacy of current research measurement methods, and did not intend to extend alternative definitive measurement methods, even a very small sample of establishments was useful in this endeavor.

\subsection{Establishment Assessments}

Visits to these establishments occurred from September 2008 through November 2009. The current research incorporated an assessment that identified nuances of the establishment's atmosphere or environment. Assessments took place on Thursday, Friday or Saturday nights, between the hours of about 7 pm and $1 \mathrm{am}$. These assessments were obtained on these "weekend" nights and during peak bar hours in order to obtain a more accurate assessment of those establishments that may have served as a restaurant during the day and as a bar or tavern during night hours. Because these establishments would still have "bar" atmospheres during these times, they would presumably still be vulnerable to the same threats as bars that were not open during the day (intoxicated patrons, etc.). Simple restaurants or other non-bar liquor-serving establishments should have maintained a "restaurant" atmosphere even during these peak hours.

The assessments consisted of a series of questions about the establishment, and a subjective account of the business's atmosphere by the researchers. The assessment itself was created using guidance from previous similar research from Graham and colleagues $(2006$; 2005). The full assessment can be seen in Appendix A. The questions were divided into three categories: First, the physical layout of the establishment identified the geographic layout of the establishment, the type of artwork present, any additional "activities" (such as Keno, trivia or billiards) and the general upkeep of the establishment. Second, the business atmosphere was noted through when patrons were asked for identification (or if they were), the staff structure (presence of host/hostess, wait staff, etc.), variety and complexity of food menu, payment restrictions, whether the establishment was part of a larger franchise, and whether dancing and/or music was offered as entertainment. Finally, the social atmosphere of the establishment was noted through the people in the establishment. This included the presumed age 
distribution, interaction between employees and patrons, interaction among patrons and any other unique interactions.

\subsection{Employee Interviews}

Interviews with employees of these establishments, which can be seen in Appendix B, served two purposes in this research. First, they were an additional indication of the type of particular establishments. Employee definitions are often shared with patrons, and as such can be very important in establishing the definition of any particular establishment. Thus, whether a waitress or bartender considered their establishment to be a "bar" or some other type of establishment was a critical component to this part of the study. This also gave an indication of whether the measurement of license-type appeared to be valid for a sample of such establishments.

Second, interviews with employees served to indicate whether (and when) particular establishments entered into the decision to notify police with regard to an attempted, in-progress, or completed assault. Because most traditional measurement of assaults includes official statistics, it follows that those assaults not reported to police are also not included in quantitative alcohol research. While this omission may not affect the validity of traditional measures if it were omitted from all alcohol-serving establishments consistently, a discovery that type of establishment may itself affect whether a call to police takes place could indicate significant problems in using police reports as a valid measure of assaults. While admittedly, employees are not always the only people who may call in an assault to police, they are often the party responsible for determining whether the authorities should indeed be notified.

Interviews were done in person at the establishment, while the interviewer was patronizing the establishment. In accordance with IRB mandate, consent was obtained prior to the interview. Because the employee was also on the job while engaging in the interview, they were loosely structured, asking a question or two before the employee left and attended to other patrons, and returned to answer a few more questions. Interviews were oral and notes were recorded manually. The complete interview can be seen in Appendix B. Only two employees refused to be interviewed, which resulted in the elimination of two establishments from the assessments. Interviewees were asked to be candid with their responses. Confidentiality was assured, and after they were matched with their appropriate atmosphere assessment, the name of the establishment was deleted from all datasets.

As generally with interviews, there were various complexities with employing this research method. Certainly, questions were subject to the interpretation of the individual being interviewed. In this respect, responses often varied in scope from one person to another. Additionally, the validity of interviews necessarily depends on the rapport that the interviewer develops with the person being interviewed, and the ability of the interviewer to clarify both questions and res- 
ponses. Because these interviews were done while the employee was working, sometimes clarifying questions and/or answers was difficult. In addition, because the researcher simultaneously acted as a patron of these establishments, the employee may have felt obligated to answer the questions in a particular way, in order to receive an adequate tip. An attempt was made to minimize these limitations by assuring the employee that their compensation was ensured regardless of their answers, and that their answers would be held in confidence, and by allowing the employee to refuse participation at any time. Aside from these limitations, interviews with staff of particular establishments contributed a great deal to this research on alcohol-selling establishments. Because employees were aware of and often even promoted a particular atmosphere for the establishment, their perceptions about both the type of establishment they worked in and their perceptions about when a fight warranted or would warrant a report to police was invaluable for determining the current downfalls of measurement for these variables.

\section{Results}

An examination of the various atmosphere assessments resulted in an identification of 5 distinct archetypes for on-premise liquor-serving establishments, based on similar characteristics among the physical/business/social arenas. Establishments which were identified as the same archetype also shared perceptions regarding when and if violent acts were considered "assaults" as well as if, when and how the assistance of law enforcement would be sought. A general description of each of these archetypes is presented below. Perhaps not surprisingly, the type of establishment coincided with the area of the city. Table 4 gives a breakdown of the different types of establishments in accordance with quadrant of the city.

"Neighborhood/Dive" bars tended to be relatively small spaces, mostly as stand-alone buildings close to residential neighborhoods. As noted in Table 4, these types of establishments comprised the majority of all establishments visited (approximately 35\%) and these establishments were more likely to be in the eastern two quadrants, which are older than the western area of the city. Establishments classified as neighborhood/dive bars generally had poor physical upkeep, and artwork (if there was any) tended to be stock posters or signs from liquor distributors. Many of these types of establishments tended to have flyers

Table 4. Establishment Type and Area of city.

\begin{tabular}{cccccc}
\hline & Northeast & Southeast & Northwest & Southwest & Total \\
\hline Neighborhood/Dive & $6^{*}$ & 5 & 0 & 3 & 14 \\
Family Restaurant & 3 & 3 & 2 & 3 & 11 \\
Meat Market & 0 & 0 & $5^{*}$ & 4 & 9 \\
Sports Lounge & 1 & 2 & 1 & 1 & 5 \\
Niche Bar & 0 & 1 & 0 & 1 & 2 \\
\hline
\end{tabular}


regarding neighborhood activities and events. Patrons were rarely asked for identification, either on entering the establishment or ordering alcohol. They were also more likely to be cash only or have a minimum purchase in order to use credit cards, but some still accepted local checks. They had a limited food menu, typically burgers and other sandwiches, but not a separate lunch or dinner menu, and they rarely had drink menus. These establishments were primarily locally owned (not part of a larger chain), and often the owner also worked at the establishment as staff. Typically, there were very limited staff at these locales (one to two).

Socially, these establishments tended to have a great deal of interaction among patrons, and between patrons and wait staff. Often, these establishments saw patrons sitting at the bar rather than tables, and age of patrons varied considerably, with a mix of older and younger patrons, and even children, which were sometimes present well into the evening. It was not unusual to see wait staff sharing drinks with patrons during their shift. It was clear from interactions that most of the patrons were well-known to other patrons and wait staff.

One of the establishments in this category (in the northeast quadrant) was not able to finish the interview, bringing the total number of interviewees in this category to 13. Interviewees at Neighborhood/Dive establishments noted that patrons primarily visited their establishment to "drink and socialize" with other patrons. Wait staff in these establishments noted that fights were not usual occurrences, although most had witnessed at least one fight. Most of interviewees in these establishments explained that, while there was no official policy for handling confrontations, the understood action was to try to avoid contacting police. Rather, a majority $(\mathrm{N}=9)$ of these establishments indicated that their first priority would be to get those involved in the confrontation outside or away from the bar. The other four establishments in this category suggested that altercations tended to be "handled" by other patrons or wait staff in the bar. Two establishments indicated that the reaction to fights depended on whether those involved were known in the establishment. If the staff knew the participants, they would intervene but if they did not, they would try and move the altercation out of the establishment. All of these establishments except one identified calling the police as a last resort because it would be "bad for business". This indicated that neighborhood/dive bar establishments tended to consider the consequences of calling the police in the long-term, rather than viewing the police as an immediate social control mechanism.

"Family Restaurants" was the second largest category of establishments visited, and these businesses were spread out evenly among each quadrant in the city. These types of establishments had physical layouts that tended to be much larger and "broken up" into individual tables or booths. They often had separate "bar" and "eating" areas and the physical condition of these establishments was clean and updated, with artwork and flyers featuring a mix of local events and recent features of the establishment (such as new drink or food offerings). Many of these establishments were part of national chains and the staff structure 
tended to be much more complex, with hostesses, wait staff and bartenders all holding separate roles.

Most family restaurants had elaborate menus, many with separate menus for appetizers, meals and desserts, and often additional drink menus. Closing times for these establishments varied, but over half of them closed an hour before mandated bar closing (which, at the time this research was being conducted, was 1 am). A majority of these restaurants hosted "happy hours" which had both food and drink specials, which catered to those getting off work on Thursday and Friday. Few of these establishments (two of the eleven) had additional activities such as pool or darts, and few had music, although some did have televisions.

While the bar area in these establishments encouraged more interaction between bartenders and/or wait staff than the eating areas, the social atmospheres were much more isolated among particular groups; groups rarely interacted with one another or with wait staff, other than conducting business transactions. During peak bar hours, these establishments tended to have most patrons in their 20's and 30's, but it was not unusual to see older patrons as well. A vast majority of patrons engaged in eating as well as drinking.

Interviews in family restaurant establishments revealed that very few physical altercations tended to take place in these types of businesses; only one of the interviewees indicated they had seen an altercation, which took place during a sporting event showing, and both patrons were asked to vacate the premises or law enforcement would be contacted, and both individuals left. Although altercations rarely happened, eight of the eleven establishments indicated that they had clear instructions regarding how to handle an altercation, should one take place. In most instances, this involved an immediate threat to notify law enforcement, and a follow through to contact the police. As noted by one interviewee, "We don't tolerate any kind of fights here. If anything happens, we let the cops take care of it - there is no way I am getting involved." This indicates a very distinct difference from the Neighborhood/Dive establishments, where notifying law enforcement was considered a less desirable response than "getting involved".

The establishments we identified as "Meat Markets" were quite different both physically and socially from the two establishments noted above. Nine of these types of establishments were visited during our research, all of which were in the western portion of the city. These establishments tended to be physically larger than Neighborhood/Dive establishments, and often had dance floors with a DJ, or more rarely, a live band. The establishments tended to be quite dark but had often had modern looking facades and updated artwork.

All of these establishments asked for identification when individuals came in the door, and seven of the nine establishments had discernible "security" staff either at the door carding patrons or in other areas of the business. Two of these establishments were cash only; the other seven took credit cards or cash. None took checks. While a few of these establishments offered snack type foods few 
patrons were seen eating during our visits. Patrons ordered drinks about equally from wait staff and from bartenders, and the bar area in these types of establishments was often quite crowded.

Socially, patrons appeared to range from their early twenties to forties, but the majority of patrons of these establishments seemed to be in their mid to late twenties or early thirties. While individuals tended to sit with their own "group" there was a great deal of interaction among patrons, and these interactions tended to range from friendly to overtly sexual. Because many of these establishments had dance floors, this was conducive to patron interaction, although there seemed to be additional interaction outside of the dance area.

One of these establishments refused an interview, so the accounts of the establishments and altercations from wait staff was only for eight of these businesses. In terms of types of customers, wait staff identified that most of their patrons are not "regulars" and that they tend to cater to a somewhat younger clientele. They indicated that "socialization and drinking" was the primary reason that individuals visited these establishments, in addition to engaging romantic behavior such as "scoring a date" or "finding someone to sleep with."

According to wait staff in six of the eight facilities, altercations were not unusual in these establishments, but the preferred method of handing such altercations seemed to vary among the specific businesses. All but one of the establishments indicated that the severity of the altercation would dictate how they handled the incident, and those that had security indicated that they were very good about either diffusing potentially volatile situations or at kicking people out of the facility. Interestingly, though, four of the establishments indicated that police had been summoned to their business, and all indicated a willingness to call police if they felt it necessary. While similar to neighborhood/dive establishments, calling the police was not a desired outcome, meat markets seemed generally more willing to make the call than those who worked in neighborhood/dive businesses. The reason for this may be that clientele in neighborhood/dive establishments were often "known" whereas those in meat markets were not known, or it may be due to the romantic nature of interactions in the meat markets versus the friendly convivial atmosphere in neighborhood/dive places.

"Sports Lounges" were, not surprisingly, establishments, in which the entire physical atmosphere revolved around sporting events. While only five of these establishments were visited, they were evenly spread throughout the city. Most of these establishments had televisions as their primary artwork, which were often showing a variety of sporting events. Television seemed to be the primary source of upkeep for these establishments, as most of them had seats, carpet or tables that were outdated but several large screen televisions. All of the establishments were well lit and were often decorated with a variety of sports memorabilia or pictures, along with alcohol advertisements. Four of the five establishments also had additional (competitive) activities such as billiards, darts or trivia. 
While in most of these establishments the bar was easily seen, all of them had secondary wait staff serving patrons. Two establishments asked for identification at the door during the hours visited. The other three asked for identification upon ordering. All accepted all forms of payment, although one required a minimum purchase to use a credit card. Often, wait staff were dressed in uniforms that were sport-related, either representing specific teams or more non-specific sporting attire. Three of the establishments were part of a national chain, while two were locally owned. The menus at all of these establishments were limited, with most offering burgers, wings, French fries and other quick fare. Three of these businesses also offered a variety of different beers, and most offered additional drink specials that coincided with sporting events.

Individuals patronizing sports lounges were overwhelmingly young males in their twenties, although a few older males and females were present in all locales. Typically, a variety of sporting events were broadcast simultaneously. Patrons, like staff, often were wearing apparel that celebrated specific sporting teams, and were often grouped near other patrons supporting the same team. Atmospheres in these businesses tended to be convivial and primarily friendly, with patrons easily interacting with one another, especially regarding the sporting events they were watching.

Wait staff reported that, while there were often verbal confrontations, physical fights in these establishments were rare. This was a universal sentiment, despite all interviewees of these establishments having witnessed at least one fight on the premises. It was interesting that the perception that fights were rare in these establishments seemed to contradict the stories regarding fights that all wait staff in these businesses relayed. Indeed, the amount of physical altercations in these types of establishments, although perceived as "rare" seemed actually to be common, although not severe. All of these wait staff indicated that they could easily predict when patrons would become out of hand, and, similar to the neighborhood/dive establishments, other patrons and staff typically would "jump in and help get them under control." Also similar to neighborhood/dive establishments, the primary response to altercations that could not be broken up was to ask patrons to leave.

Finally, two of the establishments we visited differed drastically than the other establishments, so that we identified them as their own category. These were identified as "Niche Bars" because each of them focused on filling a particular niche for patrons which fell outside the realm of the other establishments. One of these businesses specialized in organic food and drinks, with an emphasis on locally grown fare, while the other featured local artists - primarily blues and jazz performers.

These establishments were uniquely designed physically, with an emphasis on highlighting their specialty. Both had artwork from local artists and featured flyers and updates about local news and events. One of the establishments took local checks but required a minimum purchase for credit cards. The other accepted all forms of payment. 
Socially, these establishments tended to have some "regulars" but also a variety of non-regular patrons. Ages in these establishments varied widely, from early twenties to sixties. Many patrons were in their 30's and 40's. The atmospheres in both establishments were very relaxed, and it was not unusual for patrons to engage in conversation with one another or with wait staff. Wait staff in both establishments reported that they had never had problems with fights in their establishments, but that they would most likely call police right away to handle altercations. Much like the family restaurants, wait staff here indicated that fights would not be tolerated because it would detract from the atmosphere the establishment worked hard to maintain.

Clearly, these establishments differ drastically from one another regarding both the type of establishment they consider themselves to be, as well as in their criminogenic attributes. What's more, these assessments and interviews revealed that not only measurement of the establishments themselves through liquor license is imprecise at best, but also that measurement of crimes through police reports may also be inexact for some establishments. Undoubtedly, the small number of establishments assessed cannot accurately suggest a more accurate measuring tool, but the extreme variance even in such few businesses is quite telling with regard to quantitative measurement for this area of research.

Based on interviews and assessments, 21 of 38 of the establishments were identified to be highly criminogenic-12 "Neighborhood/Dives", 6 "Meat Markets" and 3 "Sports Lounges". Map 2 gives the spatial location of these establishments with the same hot spots of felonious assaults. Using these qualitative establishment definitions along with the same quantitative measure of assaults, it is appears that identifying only criminogenic establishments using qualitative methods more accurately coincided with police reports of assault. Because there were so few establishments identified, however, further statistical analyses were not completed. Nevertheless, the spatial locales of these alcohol serving establishments in relation to hot spots of assaults seems to suggest that more carefully specifying establishments using qualitative approaches may help to more accurately predict where assaults might occur.

\section{Discussion}

This research offered a critical look at the non-specific measurements often used in research on alcohol-serving urban structures. While the qualitative portion of this research, which offered a critical look at these measurements, was not extensive enough (nor was it intended) to replace those measurements, it did give an indication of where traditional measures, particularly of on-premise liquorserving establishments, fall short, and where qualitative research may enhance traditional quantitative measurements.

First, and perhaps not surprisingly, it was clear that particular types of liquorserving establishments tended to cluster in specific quadrants of the city, suggesting that the broader community culture plays an important role on estab- 


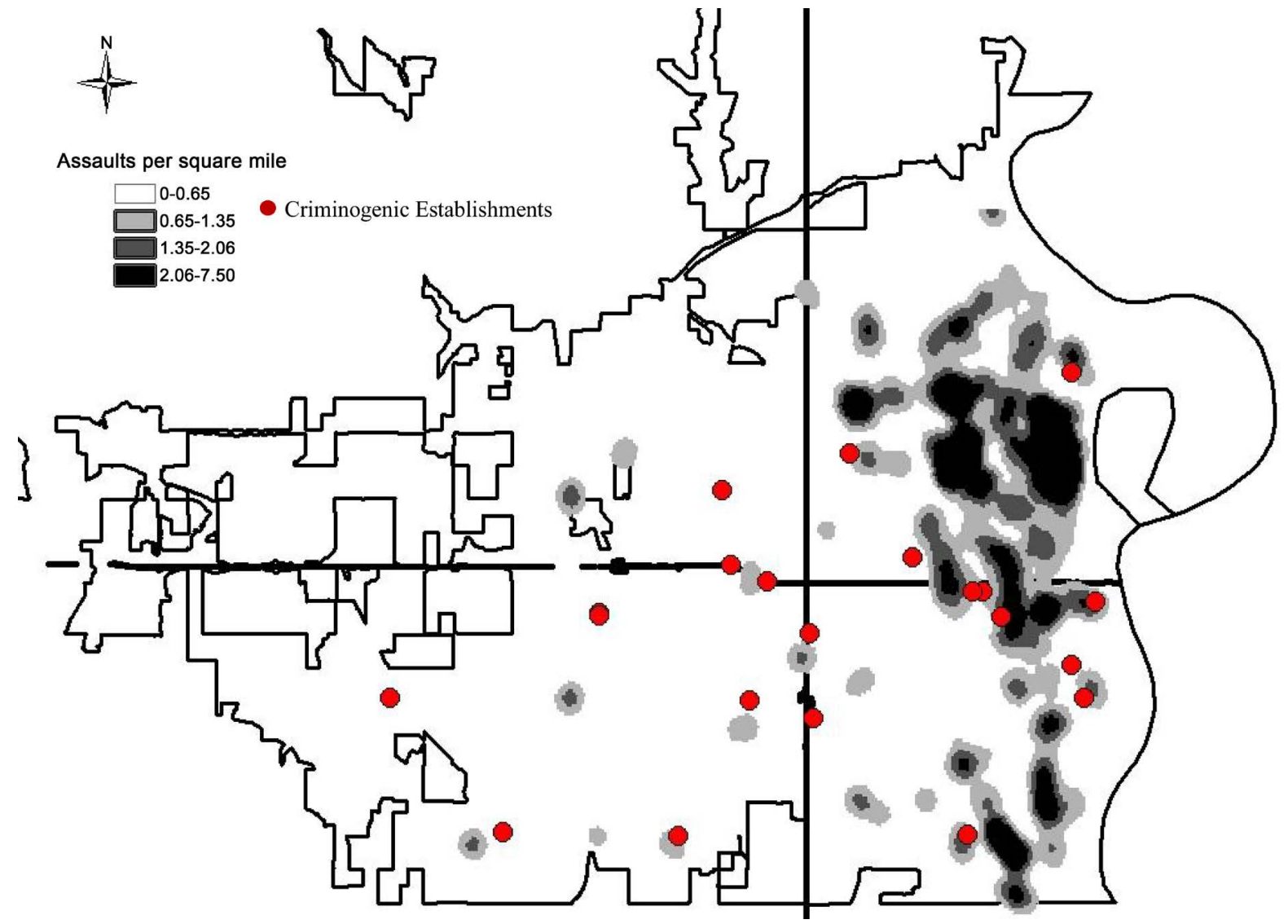

Map 2. Hot Spots of Reported Assaults (2009) and Criminogenic Establishments.

lishment atmosphere, and suggests that a standard measurement for all establishments in a city may be problematic. Another related finding is that establishments classified themselves and were classified by the researcher, as being different from other establishments that served alcohol. This research shows that, even with a very small sample, there was still variability in the type of establishment each of these businesses claimed to be. Perhaps most importantly, this research also found that the type of establishment also played into employee definitions of assaults. This means that the measurement of assaults (as a dependent variable) varies in accordance with whether an establishment views itself (and is viewed as) one of the archetypes noted in this research. Finally, this research indicated that better classification of alcohol serving establishments through qualitative techniques could better predict the likelihood of an assault occurring and being reported to the police.

These definitions are extremely crucial in light of bar research as a test of Routine Activity Theory. Not only does this research indicate that traditional macro-level-only quantitative methodology must rely on untested assumptions regarding the interpersonal mechanisms suggested by the theory, but it also indicates that results of theory testing using this methodology may differ substantially than that conducted using a micro-level qualitative or mixed-methods approach. 


\section{Conclusion}

There are several possibilities for alternative measurements for type of on-premise liquor-serving establishments, aside from physically visiting every establishment (impossible for much research, especially in larger cities). Because employee definition coincided in every case with the atmosphere assessment, this definition could be taken into account in determining whether the establishment is a bar, a restaurant or some other types of establishment. One straightforward way to determine employee definition is how they choose to list themselves in various directories. That is, whether they are advertised as a "tavern" "bar" "lounge" or "restaurant" either online or in a phone directory, could be more important than the liquor license they possess. There are practical ways to determine the type of establishment a liquor-serving business best approximates.

This research indicates that the type of on-premise liquor-serving establishment a business is might enter into how they define (and report) the specific crime of assaults. While the limitations of sample size certainly mute this finding, this research provides enough evidence that liquor serving establishments differ in their reporting of assaults. As such, the current research supports and encourages further exploration of this phenomenon. If, in fact, this finding held true for a larger sample, it may indicate that previous research is not only overestimating the number of criminogenic alcohol serving establishments, but also underestimating the number of assaults that occur there. In fact, the significant but relatively small effect of these establishments on assaults shown in the quantitative portion of this research might have been much more prevalent if a more appropriate measure of bars, assaults (or both) were used.

Although this research did, as expected, uncover the existence of a difference in definitions of establishments and assaults, setting up a definitive measure of either for future research will most certainly be difficult at best. Perhaps the greatest limitation of the current research is that it did not offer a practical alternative measurement for assaults. After all, determining whether an assault occurred based on the definitions held by those in a particular establishment at a particular time would involve interviews with all establishments. However, there may be some promising incorporation of newer techniques. Knowing the area of town in which particular types of establishments cluster, for instance, may allow the creation of clustering algorithms that might better identify geographic properties.

Although this research does not look at enough cases to warrant extensive changes in policing efforts or security measures of liquor-selling establishments, it may give an idea of which establishments may need more security or support. If, through traditional measurements, research indicates a need for greater security or support for liquor-serving establishments, but is not specifying particular types of liquor-serving establishments, it is possible that security and/or support is being overused on those establishments whose atmospheres are not conducive to violent crimes such as felonious assaults, and under-used for those establish- 
ments whose atmospheres are much more conductive to these types of behaviors.

Finally, the most important result of the current research is that is spurs similar research endeavors that continue to look at the problems with, and potential enhancements of, measurement in traditional alcohol research as a test of Routine Activity Theory. Alternative measures for both bars and assaults that retain quantitative spatial analysis but that include qualitative components should be developed and compared to the traditional measurements. Other measurement issues, such as those used to determine dispersion effects, should also be closely examined. \& certainly, replication of the current research on a larger scale is very important. As measurement techniques increase at a rapid pace in criminal justice research, assessing their validity in theory testing becomes even more crucial for their true benefit to be realized.

\section{References}

Buddie, A., \& Parks, K. A. (2003). The Role of the Bar Context and Social Behaviors on Women's Risk for Aggression. Journal of Interpersonal Violence, 18, 1378-1393. https://doi.org/10.1177/0886260503258029

Bye, E. K. (2007). Alcohol and Violence: Use of Possible Confounders in a Time-Series Analysis. Addiction, 102, 369-376.

Cohen, L. E., \& Felson, M. (1979). Social Change and Crime Rate Trends. American Sociological Review, 44, 588-608. https://doi.org/10.2307/2094589

Corbin, W. R., Bernat, J. A., \& Calhoun, K. S. (2001). The Role of Alcohol Expectancies Andalcohol Consumption among Sexually Victimized and Non-Victimized College Women. Journal of Interpersonal Violence, 16, 297-311. https://doi.org/10.1177/088626001016004002

Costanza, S. E., Bankston, W. B., \& Shihadeh, E. (2001). Alcohol Availability and Violent Crime Rates: A Spatial Analysis. Journal of Crime and Justice, 24, 71-83. https://doi.org/10.1080/0735648X.2001.9721617

Cunradi, C. B., Mair, C., Ponicki, W., \& Remer, L. (2011). Alcohol Outlets, Neighborhood Characteristics and Intimate Partner Violence: Ecological Analysis of a California City. Journal of Urban Health, 88, 191-200. https://doi.org/10.1007/s11524-011-9549-6

Felson, M. (2002). Crime and Everyday Life. Thousand Oaks, CA: Sage.

Gau, J. M. (2010). Basic Principles and Practices of Structural Equation Modeling in Criminal Justice and Criminology Research. Journal of Criminal Justice Education, 21, 136-151. https://doi.org/10.1080/10511251003693660

Gorman, D. M., Li, Z., \& Horel, S. (2005). Drug "Hot-Spots”, Alcohol availability and Violence. Drug \& Alcohol Review, 24, 507-513.

Graham, K., Bernards, S., Osgood, D. W., \& Wells, S. (2006). Bad Nights or Bad Bars? Multi-Level Analysis of Environmental Predictors of Aggression in Late-Night Large-Capacity Bars and Clubs. Addiction, 101, 1569-1580.

Graham, K., Bernards, S., Osgood, D. W., Homel, R., \& Purcell, J. (2005). Guardians and Handlers: The Role of Bar Staff in Preventing and Managing Aggression. Addiction, 100, 755-766.

Greenfield, T. K., Yu, Y., Nayak, M. B., Bond, J., Kerr, W. C., \& Trocki, K. (2011). Heaviest Drinking Locations and the Most Drunk There Predict the Likelihood of Fighting 
and Being Assaulted. Contemporary Drug Problems, 38, 213-236.

https://doi.org/10.1177/009145091103800203

Groff, E. (2011). Exploring "Near": Characterizing the Spatial Extent of Drinking Place Influence on Crime. Australian \& New Zealand Journal of Criminology, 44, 156-179. https://doi.org/10.1177/0004865811405253

Hughes, K., Anderson, Z., Morelo, M., \& Bellis, M. A. (2008). Alcohol, Night Life and Violence: The Relative Contributions of Drinking before and during Nights out to Negative Health and Criminal Justice Outcomes. Addiction, 103, 60-65.

LeSage, J. P. (2002). Short Course on Spatial Econometrics Lecture Notes. PhD Topics Lectures, Toledo: Spatial Econometrics University of Toledo. http://www.econ.utoledo.edu/faculty/lesage/papers/short_course.pdf

Mitchell, I. J., Rutherford, V., Wrinch, K. A., \& Egan, V. (2008). Paradoxical Effects of Alcohol Intake in a Convivial Social Setting. Addiction Research and Theory, 16, 503-513. https://doi.org/10.1080/16066350802113213

Nagin, D. S., \& Piquero, A. R. (2010). Using the Group-Based Trajectory Model to Study Crime over the Life Course. Journal of Criminal Justice Education, 21, 105-116. https://doi.org/10.1080/10511251003693637

Parker, R. N. (1995). Alcohol and Homicide: A Deadly Combination of Two American Traditions. Albany NY: State University of New York Press.

Quigley, B., Leonard, K. E., \& Collins, R. L. (2003). Characteristics of Violent Bars and Bar Patrons. Journal of Studies on Alcohol, 63, 765-772. https://doi.org/10.15288/jsa.2003.64.765

Ratcliffe, J. H. (2005). Detecting Spatial Movement of Intra-Region Crime Patterns over Time. Journal of Quantitative Criminology, 21, 103-123. https://doi.org/10.1007/s10940-004-1789-0

Spring, J. W., \& Block, C. R. (1988). Finding Crime Hot Spots: Experiments in the Identification of High Crime Areas. In 1988 Annual Meetings. Minneapolis, MN: Midwest Sociological Society.

U.S. Bureau of the Census (2010). U.S. Census of Population. Washington DC: Government Printing Office. 


\section{Appendix A: Establishment Atmosphere Assessment}

Physical Atmosphere:

What is the setup of the establishment (where is the bar located; where are tables, chairs, etc. located, where do people enter and what is the first item past the door)?

What sort of artwork is present in the establishment? What messages does it seem to convey?

What sorts of activities are available in the establishment (pool, darts, trivia, etc.)?

What kind of physical upkeep does the establishment seem to have (is it shoddy, clean, new, etc.)?

Business Atmosphere:

When are individuals asked for identification (or are they) for proof of age?

Describe the staff structure (are there hostesses, waitresses and waiters distinct from the bartenders, or is the structure less complex)?

Describe the selection of food and drinks offered (is there a limited menu, or a complex menu, with separate entrees for lunch and dinner)? Is there a separate drink menu?

Are there specific payment restrictions (such as no checks or cards, cash only, etc.)? How was the check presented (i.e., in a folder or simply as a receipt)?

Is the establishment locally owned, or is it a chain or franchise?

Does the establishment offer dancing? If so, what type? (i.e., patrons with each other, employees on a stage, "unofficial" dancing, etc.)

Does the establishment offer music or other entertainment distinct from patron participation?

\section{Social Atmosphere:}

What is the age distribution of patrons at the establishment (average age, as well as distribution - are most patrons within one age group? Are there children present?)

Describe the interaction between patrons and employees (do they seem to know each other well, act friendly, flirty, etc.)? 
Describe the interaction among patrons (do the interactions focus on romance, convivial friendliness, or sympathetic ears? Is there any tension or aggression?)

Discuss any additional interactions (such as between employees, among security and patrons, or particular encounters of interest).

\section{Appendix B: Questions for Wait Staff}

Type of Establishment:

What hours do you normally work?

Who are your primary customers? What age, gender, race/ethnicity? Do you have "regulars" that come in to see you? If yes, are most of your customers regulars?

Why would you say people come here on weekends and in the evening? Do people come here because they know each other and want to hang out, or to meet new people? To drink, eat, or do some other activity?

Definition of Incidents:

Have you ever seen a fight break out? How have you or would you handle a fight?

If you felt that an argument is about to escalate into a physical confrontation, what would you do?

Does your establishment have certain policies (i.e. call the police) that you follow if a fight does break out or do you try to resolve the conflict on your own?

What factors would affect how you handle a fight?

Do you think that other establishments like yours deal with fights? Why or why not? 